\title{
Liter per Hour per Milligram per Kilogram per Day
}

National Cancer Institute

\section{Source}

National Cancer Institute. Liter per Hour per Milligram per Kilogram per Day. NCI

Thesaurus. Code C120742.

A unit of concentration equal to liter per hour divided by milligram per kilog ram per day. 